Case Report

\title{
Case report of sudden death after a gunshot wound to the C2 vertebral bone without direct spinal cord injury: Histopathological analysis of spinal-medullary junction
}

\author{
Roberto Demontis ${ }^{\mathrm{a}, * *}$, Ernesto d'Aloja ${ }^{\mathrm{a}}$, Cristina Manieli ${ }^{\mathrm{b}}$, Antonio Carai ${ }^{\mathrm{a}}$, Marianna Boi ${ }^{\mathrm{c}}$, \\ Maria Pina Serrac ${ }^{\mathrm{c}}$, Marina Quartu ${ }^{\mathrm{c}, *}$ \\ a Department of Medical Sciences and Public Health, University of Cagliari, Italy \\ b Service of Pathological Anatomy, Azienda Ospedaliera “G. Brotzu", Cagliari, Italy \\ ${ }^{\mathrm{c}}$ Department of Biomedical Sciences, Section of Cytomorphology, University of Cagliari, Italy
}

\section{A R T I C L E I N F O}

\section{Article history:}

Received 7 March 2019

Received in revised form 2 June 2019

Accepted 9 June 2019

Available online 14 June 2019

\section{Keywords:}

Gunshot wound

Human medulla oblongata

Concussion injury

Shock wave-induced nervous injury

Histological analysis

Immunohistochemistry

\begin{abstract}
A B S T R A C T
Gunshot wounds (GSW) are one of the most common causes of penetrating spinal injury, however few data are available regarding GSW causing an indirect fatal nervous tissue injury, such as that induced by the concussive force secondary to the bullet penetration. This report describes a rare case of a death following a GSW spine injury at the level of C2 vertebral body, without direct contact with the spinal cord, as seen with computed tomography scan performed soon after the death. At autopsy, vertebral canal and dura mater, as well as spinal cord and medulla oblongata, appeared devoid of pathologies and/or lesions, major viscera were unaltered. The cause of death was attributed to a cardiorespiratory arrest subsequent to the GSW injury of the C2 vertebral bone. Histopathological analysis of spinal cord and medulla oblongata was performed by means of conventional stainings, and glial fibrillary acidic protein (GFAP) and Neurofilaments $200 \mathrm{kD}$ (NF) immunohistochemistry. Histological alterations stood out against a tissue with no other evident sign of neuropathology, and could be observed from the caudalmost part of the medulla oblongata to the level of the inferior olivary nucleus. Main structural changes were found in the white matter, involving often the adjacent gray matter, where they appeared as multiple scattered areas of degeneration, lacking the usual staining affinity, and showing a disrupted fibrillary pattern as evidenced by myelin staining, and GFAP- and NF-immunolabelling.

The shock wave secondary to the impact on the $\mathrm{C} 2$ vertebral bone is likely to have been the cause of a widespread neuronal-axonal histopathological damage at the spinal-medullary junction and caudal medulla oblongata that is compatible with a severe fatal respiratory dysfunction and dysregulation of the autonomic pathways subserving the control of blood pressure and cardiac activity.
\end{abstract}

(C) 2019 Published by Elsevier B.V.

\section{Introduction}

Gunshot wounds (GSW) account for about $15 \%$ of spinal injuries in the civilian population and are the most frequent cause of acute

\footnotetext{
* Corresponding author at: Department of Biomedical Sciences, Section of Cytomorphology, University of Cagliari, Cittadella Universitaria di Monserrato, 09042, Monserrato, Italy.

** Corresponding author at: Department of Medical Sciences and Public Health, Ospedale San Giovanni di Dio, University of Cagliari, Cagliari, Italy.

E-mail addresses: demrob@unica.it (R. Demontis),

ernestodaloja@medicina.unica.it (E.d'Aloja), cristinamanieli@aob.it (C. Manieli), carai@unica.it (A. Carai), marianna.boi@unica.it (M. Boi), mpserra@unica.it (M.P. Serra), quartu@unica.it (M. Quartu).
}

spinal injury [1,2]. Critical factors accounting for the different neurological upshots include the mode of injury, such as the type of weapon, the bullet properties, the firing distance, and the GSW mechanism [3]. The correlation between spinal damage and either the direct injury and/or vascular injury leading to ischemia has been extensively demonstrated in both civilian and military population [1-3]. By contrast, indirect injury from the concussive force due to the bullet in absence of vertebral bone fracture is a rare event [2]. The correlation between traumatic event and sudden death also affects other anatomical sites and contexts. As an example, the commotio cordis is a rare condition in which sudden death is triggered by a blow/trauma to the chest that causes electrophysiological changes in the heart rhythm, without evident anatomical lesions, and is exhaustively treated in the medical and 
forensic fields $[4,5]$. On the other hand, the direct connection between the commotio cerebri and the sudden death, in both the military and civilian field, would not seem to have a clear recognition as an entity in the medical-scientific literature. In fact, only few case studies give evidence of an immediate, unpredictable death due to blunt force injury in the occipito-cervical region [6-8] or to the energy transferred by a bullet on impact in the vertebral bones [2]. We report a case of an adult man who died following the entry of a missile, shot anteriorly to the mental region below the left lower lip, and finally lodged in the $\mathrm{C} 2$ body. Autopsy revealed no gross anatomical evidence of either spinal cord or brainstem injury. On the basis of the medico-legal survey and the histopathological findings we assumed that the transmission of a concussive force generated a neurogenic shock at the junction of the spinal cord and medulla oblongata, likely causing a severe dysfunction of the autonomic nervous system.

\section{Case report}

\subsection{History}

The decedent was a 47 years old civilian male, arrived on medicalized ambulance (MA) to the emergency room at 16:15 p.m. The paramedics reported that the man was shot in the head. No cardiopulmonary resuscitation was performed during transport to the hospital. The objective inspection of the corpse confirmed a compatible gun shot wound (GSW) with an entry hole $2 \mathrm{~cm}$ below the left side of the lower lip. A ragged wound of the head in the left paramedian frontal region, not compatible with the exit hole of the bullet, was also detected. The pupils were midiatric, areflexic. The oral cavity was flooded with blood; after aspiration, a lacerated lesion of the tongue left margin and the destruction of the left dental hemiarcade. Other obvious lesions of the head, trunk and limbs were not appreciated. Electrocardiogram, general examination, skull radiography and computed tomography (CT) scan of the head were carried out. Report of CT scan imaging of the head was "Presence of a metallic bullet in the soma of $\mathrm{C} 2$, with multiple bone fragments. The tip of the bullet is at the level of the cortical bone of the posterior vertebral wall, and the dura mater is displaced posteriorly and compresses the upper part of the spinal cord. Small bubbles are evident in the anterior epidural space." (Fig. 1). The final hospital report was that of a cardiorespiratory arrest due to the gunshot wound of the face with C2 vertebral injury. Because of the homicidal nature of the death, medico-legal autopsy was ordered by the Magistrate to provide time since death, and cause and circumstances of death.

\subsection{Autoptic examination}

Medico-legal autopsy was performed $43 \mathrm{~h}$ post-mortem and established that the death occurred immediately after a gunshot wound due to a calibre 7.65 bullet. The body presented no significant external injury except for the wound entry due to the impact of the projectile, with a kinetic energy compatible with a firing distance of one meter, localized in the mental region below the left lower lip. The bullet then passed through the whole anterior-posterior extension of the tongue, teared the posterior wall of the pharynx, and finally yawed and penetrated the bone tissue of the $\mathrm{C} 2$ body remaining trapped in it, fracturing the posterior wall of the bone of the vertebral body with no direct violation of the vertebral canal. No dislocation of the high cervical spine was present. The spinal cord and medulla oblongata had their normal consistency and were free of contusion.

Anterior neck dissection revealed a hemorrhagic infarction of the superficial tissues originating in the region of the right mandibular angle; after removal of muscles and other structures of
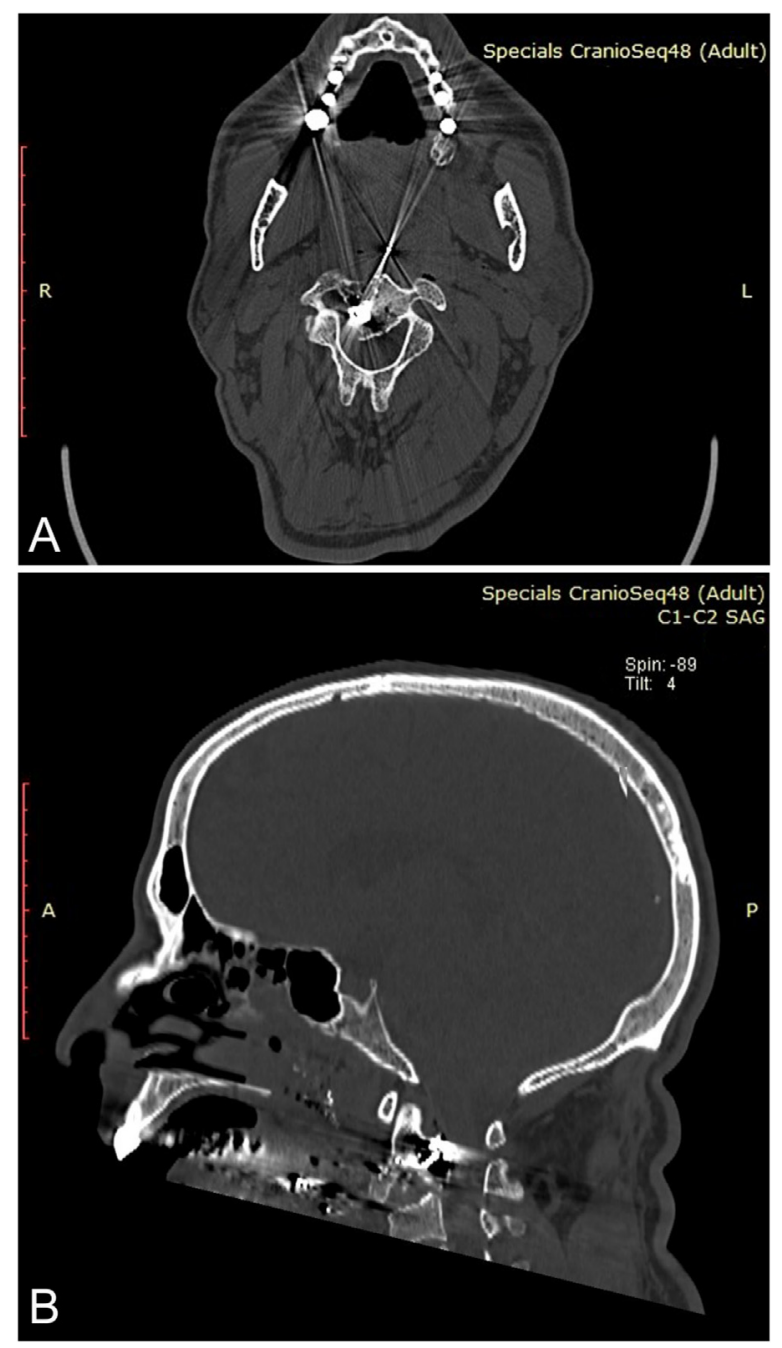

Fig. 1. Computed tomography scan in transverse (A) and sagittal frames (B) at C1C2 level.

the neck, the hemorrhagic infarction appeared to reach the retropharyngeal soft tissues, adjacent to the cervical vertebral bodies. The hemorrhagic infarction had a shape of an arrowhead with a cranial tip, wherein the retro-pharyngeal tissues appeared dissolved. The continuous solution appeared to penetrate and deepen in the bone tissue of the $\mathrm{C} 2$ body. By exploring the lesioned bone a roundish metallic body, apparently consisting of bare lead was found in the bottom of the penetrating canal. When examining the structures of the neck, the tongue, the pharynx and vertebral column were interested by the GSW. The tongue, in its left anteriorlateral margin, showed a channel-shaped passage with irregular margins, slightly leaning from left to right and from bottom to top. The wound canal opened in the tongue dorsal surface at the apex of the lingual $\mathrm{V}$, immediately in front of the lingual tonsils. A continuous round-shaped solution was found in the upper part of the pharyngeal posterior wall which appeared in continuity with the lingual penetrating lesion. Sub-millimetric metal fragments were found in the pharynx and in the peri- and retro-pharyngeal tissues. Once the C1-C2 area was achieved, a continuous roundish solution of about $8 \mathrm{~mm}$ in diameter was identified in the $\mathrm{C} 2$ body where the metallic body above mentioned has been found. Upon bilateral hemilaminectomy, no bleeding was observed in the traumatized area. After cutting the dura mater, the spinal cord had its usual consistency; though showing a blood clot on its right ventral surface, it was free of contusive areas or haemorrhagic 


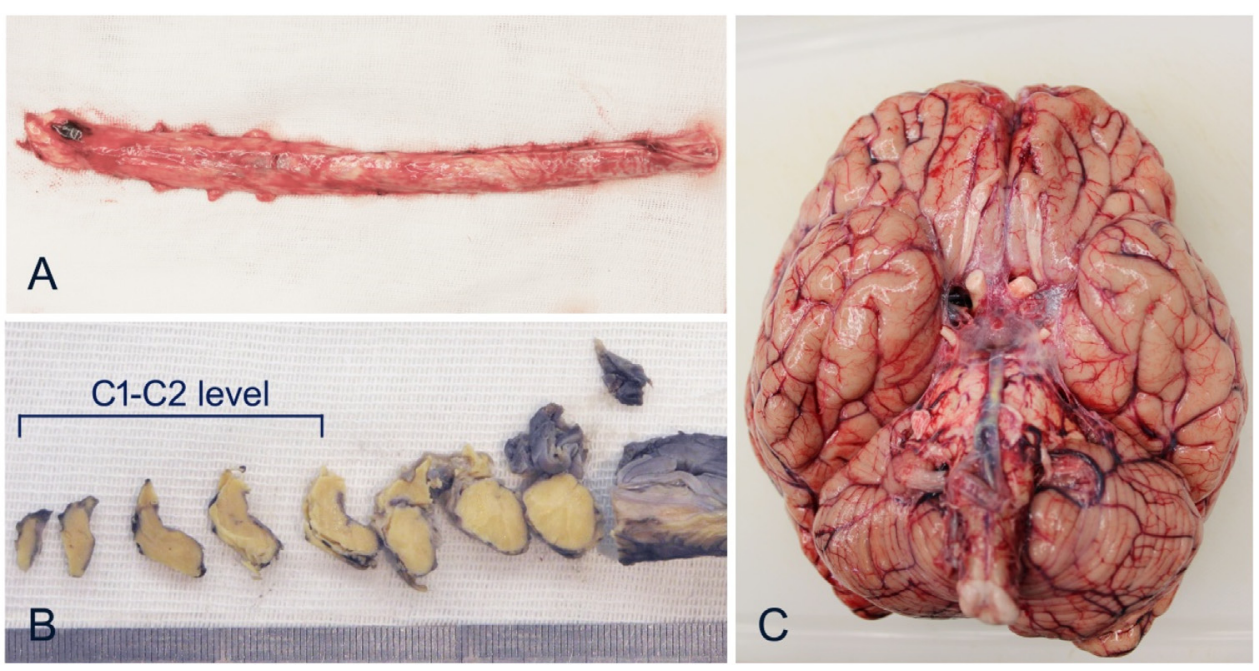

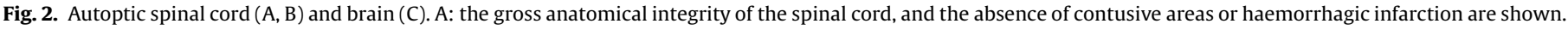
$B$ : thick serial transverse sections of cervical neuromeres showing normal consistency of the spinal cord. C: normal brain appearance.

infarction, thus appearing grossly undamaged. The outline of the gray substance and the emergence of the spinal roots and nerves were regular, as seen in fresh tissue (Fig. 2A) and after serial sectioning of the spinal cord prior to paraffin inclusion (Fig. 2B). At the scalp detachment, at the level of the pericranial tissues of the frontal median and right parietal-occipital regions two areas of hemorrhagic infiltration were present; however, the cranial bones were undamaged. After removing the cranial vault, the dura mater appeared normotensive. The brain had a regular shape with symmetrical hemispheres, and normally represented convolutions and grooves. The brain ventral surface, the Willis circle, the brainstem and the cerebellum were regular (Fig. 2C). After cutting the brain according to the Virchow technique, the ventricular cavities appeared to contain a very modest quantity of serous liquid; the choroid plexuses were congested; everywhere the limit between white and gray substance was respected and the basal ganglia showed regular boundaries. At the opening of the thoracoabdominal cavity gross pathological observation revealed that major viscera appeared normal. Weights of major viscera are reported in Table 1. Microscopical examination of the heart, lungs, liver, kidneys, and spleen revealed no histopathological alteration.

\section{Materials and methods}

Serial sections of the upper cervical spinal cord and medulla oblongata were processed for the histological and immunohistochemical analysis. The sampling and handling of human specimens conformed to the local Ethics Committee of the National Health System in compliance with the principles enunciated in the Declaration of Helsinki. The medulla oblongata, through a median sagittal cut, was sagittally separated in two halves and fixed by immersion in $4 \%$ freshly prepared phosphate-buffered

Table 1

Organ weights.

\begin{tabular}{lll}
\hline Organ & Weight, g & Range, g [9,10] \\
\hline Brain & 1388 & $1070-1767$ \\
Heart & 287 & $188-571$ \\
Right lung & 610 & $185-967$ \\
Left lung & 530 & $186-885$ \\
Spleen & 165 & $43-344$ \\
Liver & 1430 & $945-1689$ \\
Right kidney & 147 & $79-223$ \\
Left kidney & 158 & $74-235$ \\
\hline
\end{tabular}

formaldehyde, $\mathrm{pH} 7.3$, for $4-6 \mathrm{~h}$ at $4^{\circ} \mathrm{C}$. One half was cut with a cryostat in consecutive sections ( $16 \mu \mathrm{m}$ thick) sections, the other was processed for paraffin inclusion and cut with a microtome in 6 $\mu \mathrm{m}$ sections. The slices were deparaffinised with xylene, rehydrated in graded concentrations of ethanol prior to Haematoxylin and Eosin (HE), Masson trichrome, Luxol Fast Blue (LFB) and Klüver-Barrera method (KB), used as histological, Nissl and myelin stainings [11], and to immunohistochemistry through an automated Leica Bond III (LeicaMicrosystems, Melbourne, Australia) using a Bond Polymer Refine Detection system (Leica Biosystems, Newcastle, UK). Ready to Use antibodies against glial fibrillary acidic protein (GFAP) (clone GA5), synaptophysin (clone 27G12), and Neurofilaments $200 \mathrm{kD}$ (NF) (clone N52.1.7) were used as primary antibodies. Heat-Induced Epitope Retrieval (HIER) was performed in Citrate-based buffer, pH 5-6, for $5 \mathrm{~min}$, in the case of GFAP and NF, and in Ethylenediaminetetraacetic acid (EDTA), pH 9, for $20 \mathrm{~min}$, in the case of synaptophysin. Negative control preparations were obtained by omitting the primary antibody. All slides were dehydrated and coverslipped. Observations and photographs were made with a photomicroscope Olympus BX61 (Hamburg, Germany) equipped with a Leica DFC450 C camera connected with the PC for the acquisition of digital images by means of LAS AF software and with Nanozoomer 2.0-RS (Hamamatsu).

\section{Results}

Histopathological analysis of serial sections of the cervical neuromeres of the spinal cord and medulla oblongata showed that histological alterations of the white matter could be observed and were limited to the level of the spinal cord/medullary transition and to the medulla oblongata. In the HE stained sections of medulla oblongata (Fig. 3A, B), multiple scattered areas of degenerative changes, with loss of the usual fibrillary pattern and decrease in staining affinity, stood out against a nervous tissue almost normal and with no obvious signs of neuropathology. In the same areas, at the boundary with the adjacent normal tissue, nervous and glial cells appeared disrupted or with cytoplasmic swelling. All these structural changes are similar, although with a scattered distribution, to the neuropathological frame induced by an early hypoxicischemic brain injury $[12,13]$. The same scattered round areas of degeneration were also highlighted after the KB myelin staining (Fig. 3C, D). The immunohistochemical reaction for GFAP showed that the fibrillary fragmentation involves the close network of 

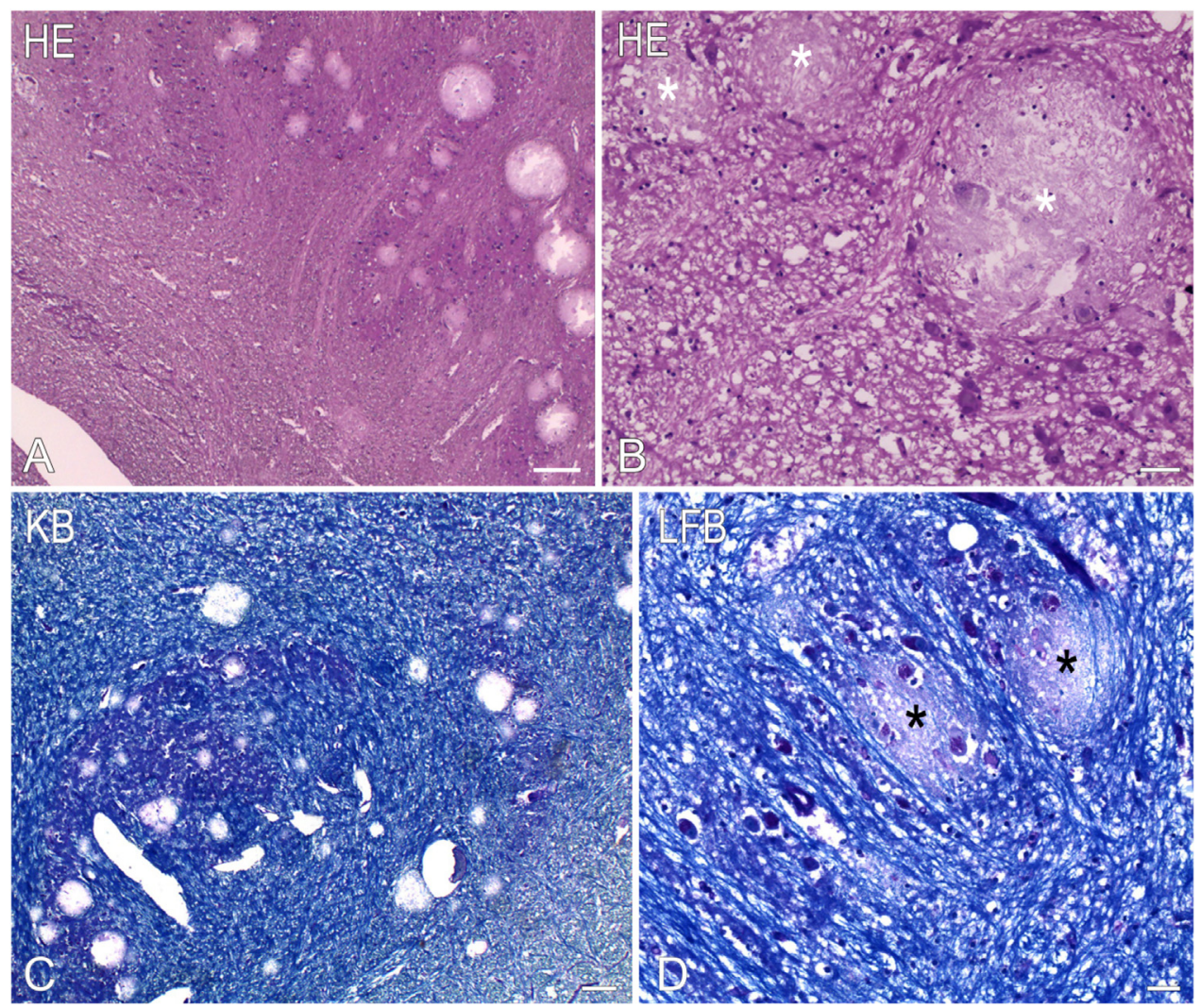

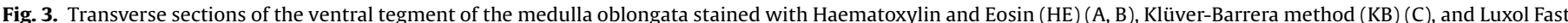

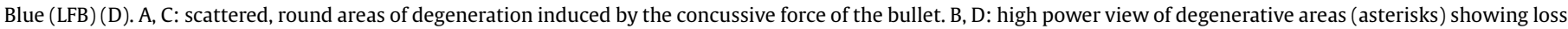
of the usual fibrillary texture and cell swelling, suggestive of acute neuronal injury. Scale Bars: $A, C=100 \mu \mathrm{m} ; B, D=20 \mu \mathrm{m}$.

astrocytic intermediate filaments (Fig. 4A, B). A neuropathological pattern of the neuropil, characterized by a loose multifragmented network of neuronal processes, and consistent with acute axonal damage, was also observed after NF-immunostaining (Fig. 4C, D).

\section{Discussion}

Spinal cord injuries as a result of gunshot wounds represent approximately the third most recurring worldwide cause of penetrating spine injury with an incidence of $13 \%-17 \%[2,14]$. GSW-induced spinal injury yields a range of different outcomes, depending on various mechanical and biological factors [2]. Most frequently the spinal cord sustains a direct trauma, such as contusion, transection, vascular lesion and ischemia, that can be associated with damage of surrounding bones and soft tissues; thus, available data largely regard the conservative management of patients who survived to GSW to the spine with subsequent contusion of spinal cord $[15,16]$. Studies regarding the pathogenesis of GSW to the spine also focus on functional and radiological aspects of the lesion in order to guide surgical and therapeutical decision-making in the specific neurological cases $[1,2,18,19]$.

Here we have reported a rare case of a 47 years old male with fatal outcome following a GSW at C2 level without frank vertebral canal violation and without a direct contact of the bullet's track with the spinal cord. Cases of spinal cord lesion following GSW cervical spine injury without canal compromise, leading however to a favourable outcome, have been reported; patients survived, few days to years, either without neural lesion but with upper airways impairment $[20,21]$ or with different degree of neurological deficits $[1,15,22,23]$. To our knowledge no similar cases have been described yet and available reports are only partially comparable since the bulk of them regards the neurological outcome in surviving patients. A unique fatal outcome due to neurogenic shock caused by concussion of the central nervous system at the level of spinomedullary junction was reported for a case regarding a 13 years old girl, suddenly deceased following a blow to the neck at the level of occipital-cervical spine [7]. In their report, Davis and Glass [7] also stated that the spinal cord had the usual consistency and did not show contusion either grossly or histologically. One case of rehabilitation of a patient with C2 spine fracture after a traffic accident appears partially comparable to the case here described; there, a hyperintensity from medulla oblongata to level C3 of spinal cord was indicated by Magnetic Resonance Imaging (MRI), and the patient transiently suffered of hyperesthesia, quadriplegia and gatism [23]. A single case of a bullet lodged in the subdural space at upper cervical region (C1$\mathrm{C} 2$ ), without any bone destruction, and without any spinal cord injury, as proved by CT scan, has been reported [15]. A further case regarding a 31 years old patient sustaining a fracture of the $\mathrm{C} 5$ spinous process due to gunshot injury to the neck, with no direct spinal cord injury, reported of unexpected delayed tetraplegia [24].

Other available retrospective reviews addressed neurological patients with GSW spinal injury at different thoracic level $[17,25]$. In 1979 , Stauffer et al. [25], by reviewing 185 patients with GSW to the spine, found that 101 out of 185 patients underwent laminectomy and 11 of them, with either complete or incomplete lesions, had a normal spinal cord. However, no further diagnostic details, such as mielography, magnetic resonance, or possible compression of the spinal cord, had been given to prove their findings. In a more recent retrospective review of patients with GSW spinal injury, regarding 3 of 26 cases with complete paraplegia at thoracic level at 4 year follow up, diagnostic imaging or surgical exploration did not show any sign of vertebral canal violation, and led to suggest that the neurologic deficit had been 

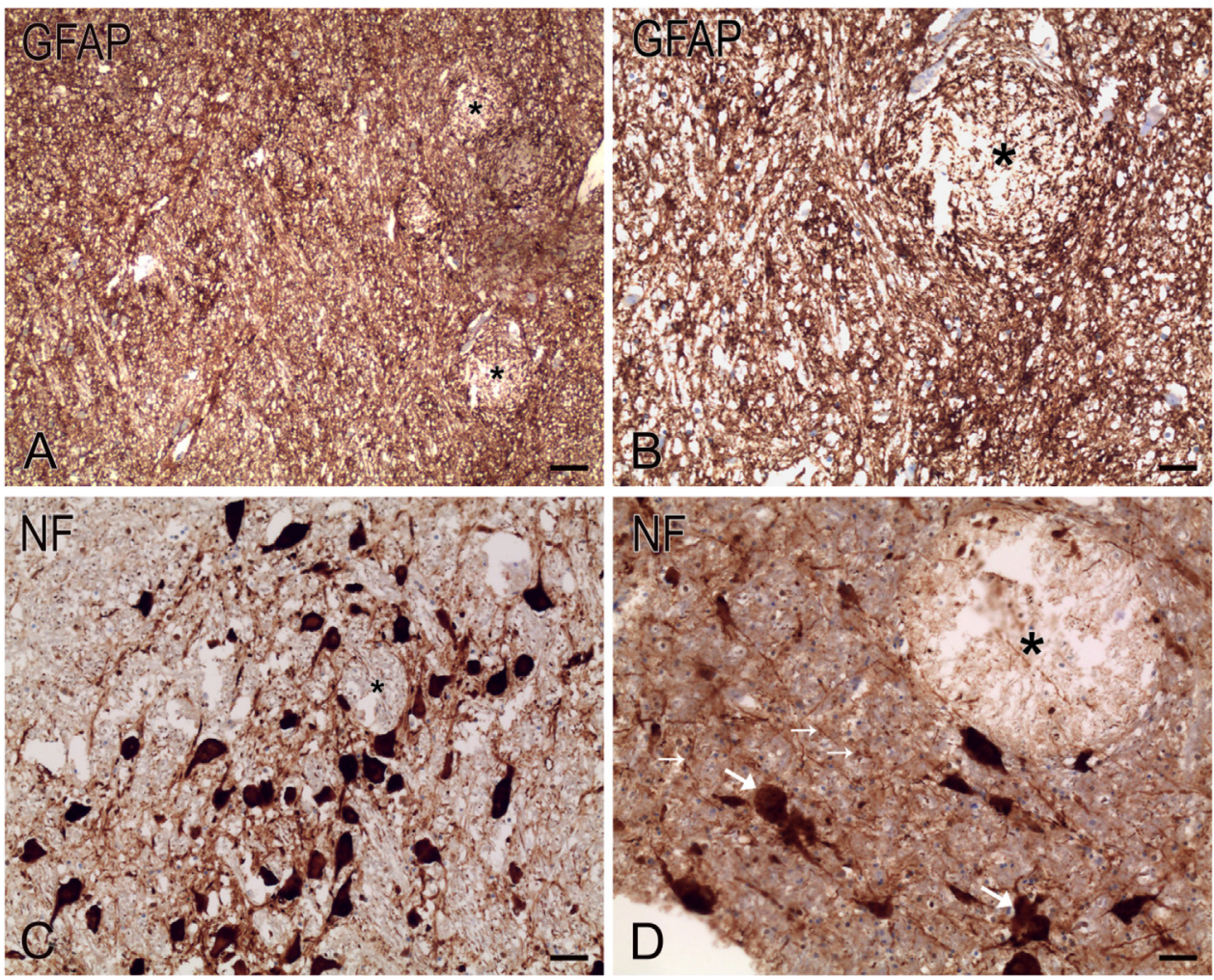

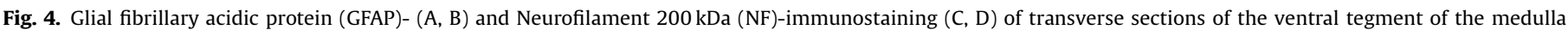

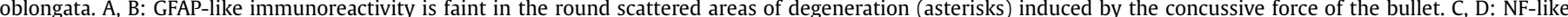

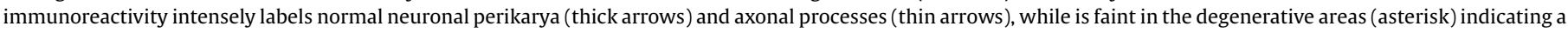
loss of the usual fibrillary texture. Scale Bars: A, C $=100 \mu \mathrm{m} ; B, D=20 \mu \mathrm{m}$.

due to the kinetic energy emitted by the bullet [17]. Patil et al. [2] reported a case of paraplegia with complete loss of sensation below D10 level as a result of GSW causing spinal cord injury without bony injuries and violation of spinal axis. A similar case of conservative management has been reported by Khan et al. [16], who showed contusion to the spinal cord, without direct trauma, in a patient with complete paraplegia after a GSW injury in the T1-T2 region.

From a ballistic point of view, the outcome of bullet interaction with the tissue is strictly related to its penetrating force and destructive effect, both of which depend upon the projectile kinetic energy. Thus, the leading edge of the bullet penetrates the tissue according to a composite mechanism provided by the triple action of hammer, i.e. the projectile crushes the contacted tissue due to the overpressure created in its proximity, wedge, i.e. the projectile diverges the surrounding tissue parts determining the so-called wound channel, and drill, that facilitates penetration and transmits to the tissues the projectile rotational movement $[3,26]$. The resultant effect is the generation of pressure (or "shock") waves, lasting milliseconds, which act on the neighbouring tissues for a distance proportional to the calibre of the projectile $[3,26,27,28]$.

On the basis of the neuropathological results, and in the absence of an evident anatomical death, we believe that the observed structural damage of the nervous tissue represents the morphological ground of the bullet-generated concussive force, even without a direct contact or a straightforward injury of the nervous tissue. Since the literature on the GSW injury to the cervical spine regards neurological patients, we can only speculate about the aspect of histopathological findings related to the present case. Several studies attempted to establish a scientific rationale behind the wound ballistics theories [[27],26,28]; thus, the remote effects seen in tissue as the result of shock waves, rather than the most frequently observed temporary cavitations, has been already prompted as the cause of nerve stimulation and histological changes upon an extremely rapid increase in pressure, followed by a sudden reduction in pressure [27 and refs in it]. Accordingly, studies on simulant animal models demonstrated that the ballistic pressure wave alone can lead to brain injury [ 27,29]. Moreover, a relationship between traumatic brain injury and ballistics pressure waves originating in the thoracic cavity and extremities has been established in neurological patients [29].

We suggest that in the present case report, an acute clinical condition of spinal shock affecting the spinal cord-medullary junction had been fatal, since the death likely occurred following a combination of a respiratory compromise and a neurogenic shock. The respiratory impairment has been shown to be sustained from trauma at any spinal segment owing to dysfunctional activity of respiratory muscles [30]; thus, the injury at high cervical levels can result in diaphragm dysfunction due to interruption of bulbospinal respiratory projections to phrenic motor neuron groups (C3-C5). The neurogenic shock involves a dysregulation of the autonomic pathways and creates a complex clinical condition characterized by loss of autonomic functions with drastic reduction of sympathetic nerve tone, blood pressure and cardiac activity, which can be determined, among others, by a clinical condition of reduced vascular supply to the spinal cord or to medullary traumatic injuries [31].

\section{Conclusion}

The shock wave secondary to the projectile impact on the body of the second vertebral bone is likely to have been the cause of a widespread neuro-axonal damage at the level of the spinal cordmedulla oblongata junction. The simple transfer of the bullet kinetic energy may have caused a fatal neurological impairment 
due to a severe respiratory dysfunction and to a dysregulation of the autonomic system controlling blood pressure and cardiac activity.

\section{Conflicts of interest}

The authors declare no conflict of interest.

\section{CRediT authorship contribution statement}

Roberto Demontis: Conceptualization, Formal analysis, Investigation, Validation, Writing - original draft. Ernesto d'Aloja: Formal analysis. Cristina Manieli: Formal analysis. Antonio Carai: Investigation. Marianna Boi: Investigation. Maria Pina Serra: Funding acquisition, Investigation. Marina Quartu: Conceptualization, Formal analysis, Funding acquisition, Supervision, Validation, Visualization, Writing - original draft, Writing - review \& editing.

\section{Acknowledgements}

The study was supported by grants from the Italian Ministry of Education,Universities and Research (Fondo per il finanziamento delle attività base di Ricerca, FFABR-F35D17000170001) and from the University of Cagliari (Fondo Integrativo per la Ricerca, FIR 2017, 2018).

\section{References}

[1] G.S. Sidhu, A. Ghag, V. Prokuski, A.R. Vaccaro, K.E. Radcliff, Civilian gunshot injuries of the spinal cord: a systematic review of the current literature, Clin. Orthop. Relat. Res. 471 (2013) 3945-3955, doi:http://dx.doi.org/10.1007/ s11999-013-2901-2.

[2] R. Patil, G. Jaiswal, T.K. Gupta, Gunshot wound causing complete spinal cord injury without mechanical violation of spinal axis: case report with review of literature, J. Craniovertebr. Junction Spine 6 (2015) 149-157, doi:http://dx.doi. org/10.4103/0974-8237.167855.

[3] P.K. Stefanopoulos, D.E. Pinialidis, G.F. Hadjigeorgiou, K.N. Filippakis, Wound ballistics 101: the mechanisms of soft tissue wounding by bullets, Eur J Trauma Emerg Surg 43 (2017) 579-586, doi:http://dx.doi.org/10.2017/s00068-015-0581-1.

[4] D.T. Marshall, J.D. Gilbert, R.W. Byard, The Spectrum of Findings in Cases of Sudden Death Due to Blunt Cardiac Trauma-'Commotio Cordis', Am. J. Forensic Med. Pathol. 29 (2008) 1-4, doi:http://dx.doi.org/10.1097/PAF.0b013e31815b4d37.

[5] L. Krexi, M.N. Sheppard, Blow/trauma to the chest and sudden cardiac death: Commotio cordis and contusio cordis are leading causes, Med. Sci. Law 1 (January) (2018), doi:http://dx.doi.org/10.1177/0025802418754961 25802418754961.

[6] C.B. Courville, Commotio Cerebri. Cerebral Concussion and the Postconcussion Syndrome in their Medical and Legal Aspects, San Lucas Press, Los Angeles, 1953, doi:http://dx.doi.org/10.1212/WNL.3.6.477.

[7] G.G. Davis, J.M. Glass, Case report of sudden death after a blow to the back of the neck, Am. J. Forensic Med. Pathol. 22 (2001) 13-18.

[8] M.K. Kasliwal, R.B. Fontes, V.C. Traynelis, Occipitocervical dissociationincidence, evaluation, and treatment, Curr. Rev. Musculoskelet. Med. 9 (2016) 247-254, doi:http://dx.doi.org/10.1007/s12178-016-9347-6.

[9] D.K. Molina, V.J. DiMaio, Normal organ weights in men: part I-the heart, Am. J. Forensic Med. Pathol. 33 (2012) 362-367, doi:http://dx.doi.org/10.1097/ PAF.0b013e31823d298b.
[10] D.K. Molina, V.J. DiMaio, Normal organ weights in men: part II-the brain, lungs, liver, spleen, and kidneys, Am. J. Forensic Med. Pathol. 33 (2012) 368-372, doi: http://dx.doi.org/10.1097/PAF.0b013e31823d29ad.

[11] M. Del Fiacco, M. Quartu, M.P. Serra, M. Boi, R. Demontis, L. Poddighe, C. Picci, T. Melis, The human cuneate nucleus contains discrete subregions whose neurochemical features match those of the relay nuclei for nociceptive information, Brain Struct. Funct. 219 (2014) 2083-2101, doi:http://dx.doi.org/ 10.1007/s00429-013-0625-4.

[12] J. Van Reempts, The hypoxic brain: histological and ultrastructural aspects, Behav. Brain Res. 14 (1984) 99-108, doi:http://dx.doi.org/10.1016/0166-4328 (84)90177-3.

[13] O. Mărgăritescu, L. Mogoantă, I. Pirici, D. Pirici, D. Cernea, C. Mărgăritescu, Histopathological changes in acute ischemic stroke, Rom. J. Morphol. Embryol. 50 (2009) 327-339 PMID: 19690757.

[14] T.E. de Barros Filho, A.F. Cristante, R.M. Marcon, A. Ono, R. Bilhar, Gunshot injuries in the spine, Spinal Cord 52 (2014) 504-510, doi:http://dx.doi.org/ 10.1038/sc.2014.56.

[15] M. Seçer, M. Ulutaș, E. Yayla, K. Cinar, Upper cervical spinal cord gunshot injury without bone destruction, Int. J. Surg. Case Rep. 5 (2014) 149-151, doi:http:// dx.doi.org/10.1016/j.ijscr.2014.01.009.

[16] K. Khan, B. Dieudonne, S. Saeed, S. Alothman, Y. Saeed, S. Gray, Paraplegia following spinal cord contusion from an indirect gunshot injury, Korean J. Neurotrauma.14(2018)32-34, doi:http://dx.doi.org/10.13004/kjnt.2018.14.1.32.

[17] Y. Mirovsky, E. Shalmon, A. Blankstein, N. Halperin, Complete paraplegia following gunshot injury whithout direct trauma to the cord, Spine 30 (2005) 2436-2438, doi:http://dx.doi.org/10.1097/01.brs.0000184588.54710.61.

[18] O.R. Hubschmann, A.J. Krieger, F. Lax, P.O. Ruzicka, A.E. Zimmer, Syndrome of intramedullary gunshot wound with incomplete neurologic deficit: case report, J. Trauma. 28 (1988) 1600-1602 PMID: 3184227.

[19] P.C. Kupcha, H.S. An, J.M. Cotler, Gunshot wounds to the cervical spine, Spine (Phila Pa 1976) 15 (1990) 1058-1063 PMID: 2263972.

[20] I. Mohamad, M.Y. Musa, A.S. Razaq, Acute upper airway obstruction secondary to gunshot injury splitting cervical vertebra, Ann. Acad. Med. Singapore 40 (2011) 430-431 PMID: 22065041.

[21] B.L. Fetterman, M.L. Shindo, R.B. Stanley Jr., W.B. Armstrong, D.H. Rice Management of traumatic hypopharyngeal injuries, Laryngoscope 105 (1995) 8-13, doi:http://dx.doi.org/10.1288/00005537-199501000-00005.

[22] W.S. Paiva, R.L. Amorim, D.F. Menendez, R.S. Brock, A.F. Andrade, M.J. Teixeira, Gunshot wound to the upper cervical spine leading to instability, Int. J. Clin. Exp. Med. 7 (3) (2014) 789-791 PMID: 24753780.

[23] J. Shi, C. Lan, C. Zhu, Z. Yi, L. Chen, C. Zhang, Rehabilitation of a SCl patient involving the medulla oblongata injury after a traffic accident: a case report, Int. J. Clin. Exp. Med. 9 (2016) 22648-22651 ISSN:1940-5901/ IJCEM0028061.

[24] S.S. Goonewardene, K.S. Mangat, I.D. Sargeant, K. Porter, I. Greaves, Tetraplegia following cervical spine cord contusion from indirect gunshot injury effects, J. R. Army Med. Corps 153 (2007) 52-53, doi:http://dx.doi.org/10.1136/jramc153-01-12.

[25] E.S. Stauffer, R.W. Wood, E.G. Kelly, Gunshot wounds of the spine: the effect of laminectomy, J. Bone Joint Surg. Am. 61 (1979) 389-392 PMID: 429409.

[26] N.E. McSwain Jr., Ballistics, in: R.R. Ivatury, C.G. Cayten (Eds.), The Textbook of Penetrating Trauma, Williams \& Wilkins, Baltimore, 1996, pp. 105-119.

[27] N. Maiden, Historical overview of wound ballistics research, Forensic Sci. Med. Pathol. 5 (2009) 85, doi:http://dx.doi.org/10.1007/s12024-009-9090-z.

[28] J. Breeze, A.J. Sedman, G.R. James, T.W. Newbery, A.E. Hepper, Determining the wounding effects of ballistic projectiles to inform future injury models: a systematic review, J. R. Army Med. Corps 160 (2014) 273-278, doi:http://dx. doi.org/10.1136/jramc-2013-000099.

[29] A. Courtney, M. Courtney, Links between traumatic brain injury and ballistics pressure waves originating in the thoracic cavity and extremities, Brain Inj. 21 (2007) 657-662, doi:http://dx.doi.org/10.1080/026990 50701481571.

[30] M.A. Lane, D.D. Fuller, T.E. White, P.J. Reier, Respiratory neuroplasticity and cervical spinal cord injury: translational perspectives, Trends Neurosci. 31 (10) (2008) 538-547, doi:http://dx.doi.org/10.1016/j.tins.2008.07.002.

[31] K.L. McCance, S.E. Huether, Pathophysiology, The Biologic Basis for Disease in Adults and Children, 8th edition, Mosby Elsevier, St. Louis, MO, 2018 ISBN 9780323583473. 